\title{
Feasibility and efficacy of lung ultrasound to investigate pulmonary complications in patients who developed postoperative Hypoxaemia-a prospective study
}

Chen Xie ${ }^{1}$, Kai Sun ${ }^{1}$, Yueyang You', Yue Ming ${ }^{1}$, Xiaoling Yu ${ }^{1}$, Lina Yu ${ }^{1}$, Jiapeng Huang ${ }^{2}$ and Min Yan ${ }^{1,3^{*}}$ (D)

\begin{abstract}
Background: Postoperative pulmonary complications (PPCS) and hypoxaemia are associated with morbidity and mortality. We aimed to evaluate the feasibility and efficacy of lung ultrasound (LUS) to diagnose PPCs in patients suffering from hypoxaemia after general anaesthesia and compare the results to those of thoracic computed tomography (CT).
\end{abstract}

Methods: Adult patients who received general anaesthesia and suffered from hypoxaemia in the postanaesthesia care unit (PACU) were analysed. Hypoxaemia was defined as an oxygen saturation measured by pulse oximetry $\left(\mathrm{SPO}_{2}\right)$ less than $92 \%$ for more than $30 \mathrm{~s}$ under ambient air conditions. LUS was performed by two trained anaesthesiologists once hypoxaemia occurred. After LUS examination, each patient was transported to the radiology department for thoracic $\mathrm{CT}$ scan within $1 \mathrm{~h}$ before returning to the ward.

Results: From January 2019 to May 2019, 113 patients (61 men) undergoing abdominal surgery (45 patients, 39.8\%), video-assisted thoracic surgery (31 patients, 27.4\%), major orthopaedic surgery (17 patients, 15.0\%), neurosurgery (10 patients, 8.8\%) or other surgery (10 patients, 8.8\%) were included. CT diagnosed 327 of 1356 lung zones as atelectasis, while LUS revealed atelectasis in 311 of the CT-confirmed zones. Pneumothorax was detected by CT scan in 75 quadrants, 72 of which were detected by LUS. Pleural effusion was diagnosed in 144 zones on CT scan, and LUS detected 131 of these zones. LUS was reliable in diagnosing atelectasis (sensitivity 98.0\%, specificity $96.7 \%$ and diagnostic accuracy 97.2\%), pneumothorax (sensitivity $90.0 \%$, specificity $98.9 \%$ and diagnostic accuracy 96.7\%) and pleural effusion (sensitivity 92.9\%, specificity $96.0 \%$ and diagnostic accuracy $95.1 \%$ ).

Conclusions: Lung ultrasound is feasible, efficient and accurate in diagnosing different aetiologies of postoperative hypoxia in healthy-weight patients in the PACU.

Trial registration: Current Controlled Trials NCT03802175, 2018/12/05, www.ClinicalTrials.gov

Keywords: Lung ultrasound, Atelectasis, Pneumothorax, Pleural effusion, Thoracic computed tomography

\footnotetext{
* Correspondence: zryanmin@zju.edu.cn

'Department of Anesthesiology and Pain Medicine, the Second Affiliated Hospital, School of Medicine, Zhejiang University, Jiefang Road 88th, Hangzhou 310016, People's Republic of China

${ }^{3}$ Department of Anesthesiology and Pain Medicine, Second Affiliated

Hospital, Zhejiang University School of Medicine, 88 Jiefang Road, Hangzhou 310009, NO, China

Full list of author information is available at the end of the article
}

(c) The Author(s). 2020 Open Access This article is licensed under a Creative Commons Attribution 4.0 International License, which permits use, sharing, adaptation, distribution and reproduction in any medium or format, as long as you give appropriate credit to the original author(s) and the source, provide a link to the Creative Commons licence, and indicate if changes were made. The images or other third party material in this article are included in the article's Creative Commons licence, unless indicated otherwise in a credit line to the material. If material is not included in the article's Creative Commons licence and your intended use is not permitted by statutory regulation or exceeds the permitted use, you will need to obtain permission directly from the copyright holder. To view a copy of this licence, visit http://creativecommons.org/licenses/by/4.0/. The Creative Commons Public Domain Dedication waiver (http://creativecommons.org/publicdomain/zero/1.0/) applies to the data made available in this article, unless otherwise stated in a credit line to the data. 


\section{Background}

Postoperative pulmonary complications (PPCs), including atelectasis, pulmonary oedema, pneumonia, etc., are common, persistent and related to poor patient outcomes, medical costs, hospital readmissions and even significant mortality [1]. Hypoxaemia is mainly caused by atelectasis and occurs frequently in the immediate postoperative recovery course in both paediatric and adult patients; hypoxaemia is also associated with nausea, vomiting, postoperative cognitive dysfunction, surgical site infection, arrhythmias, prolonged hospital stay and death [2-7].

Rapid diagnosis and appropriate management must be made by the anaesthesiologist once hypoxia occurs postoperatively. The use of chest X-rays (CXR) is limited due to the disadvantage of poor quality [8]. Although thoracic computed tomography $(\mathrm{CT})$ is considered the gold standard to elucidate the causes of hypoxia, radiation exposure and the need to transfer unstable patients make CT a less-than-ideal tool. Bedside lung ultrasound (LUS) has the advantages of sensitivity, accuracy, non-radiation, non-invasiveness, reproducibility and convenience. LUS has been validated for the diagnosis of atelectasis, pneumonia, pleural effusion and pneumothorax [9-14].

The aim of this study was to evaluate the feasibility and efficacy of LUS to diagnose PPCs in patients suffering from hypoxaemia after general anaesthesia in the postanaesthesia care unit (PACU) and compare LUS results with those of thoracic CT.

\section{Methods}

\section{Patients}

The study was approved by the review committee of the Second Affiliated Hospital of Zhejiang University (IR2018001133, 2018/12/05) and registered at ClinicalTrials.gov (NCT03802175) before patient enrolment. Informed consent was obtained from all patients. Adult patients who received general anaesthesia and suffered hypoxaemia in the PACU were included in this study. Postoperative hypoxaemia was defined as decreased oxygen saturation measured by pulse oximetry $\left(\mathrm{SPO}_{2}\right)$ less than $92 \%$ for greater than $30 \mathrm{~s}$ while under ambient air conditions $20 \mathrm{~min}$ after extubation [15]. The exclusion criteria included the following: covered surgical dressings from open thoracic or breast surgery preventing ultrasound examination; body mass index (BMI) greater than $40 \mathrm{~kg} / \mathrm{m}^{2}$; lack of cooperation due to cognitive dysfunction; residual muscle relaxants resulting in incomplete recovery of muscle strength (train-of-four stimulation, $\mathrm{TOF}<0.9$ ); respiratory forgetfulness from residual opioid; haemodynamic instability; anaemia; and significant bleeding, fever or hypothermia. In addition, patients were withdrawn if their $\mathrm{SPO}_{2}$ declined to $85 \%$ or less or if admission to the intensive care unit (ICU) occurred.

\section{Anaesthesia protocol}

Before the induction of anaesthesia, all patients were preoxygenated with an inspiration oxygen fraction $\left(\mathrm{FiO}_{2}\right)$ of 1.0 for $3 \mathrm{~min}$. Anaesthesia was induced with midazolam $0.05-$ $0.1 \mathrm{mg} / \mathrm{kg}$, sufentanil $25-35 \mu \mathrm{g}$, etomidate $0.2-0.4 \mathrm{mg} / \mathrm{kg}$ and rocuronium $0.5-0.7 \mathrm{mg} / \mathrm{kg}$. A proper double-lumen endotracheal tube was intubated to perform one-lung ventilation (OLV) during video-assisted thoracoscopic surgery (VATS), whereas a common tracheal tube was inserted for two-lung ventilation (TLV) during non-VATS. Continuous intravenous propofol, remifentanil and inhalational sevoflurane were utilized for anaesthesia maintenance after intubation. Supplemental cisatracurium was provided for adequate muscle relaxation when needed. Volumecontrolled ventilation with tidal volume of $5-8 \mathrm{~mL} / \mathrm{kg}$ (5-6 $\mathrm{mL} / \mathrm{kg}$ for OLV and $6-8 \mathrm{~mL} / \mathrm{kg}$ for TLV), respiratory rate (RR) of $12-15$ breaths/min, $\mathrm{FiO}_{2}$ of $0.5-0.6$ and positive end-expiratory pressure (PEEP) of $5 \mathrm{~cm} \mathrm{H}_{2} \mathrm{O}$ was utilized to maintain an end-tidal carbon dioxide pressure $\left(\mathrm{P}_{\mathrm{ET}} \mathrm{CO}_{2}\right)$ between 35 and $45 \mathrm{mmHg}$ and a peak airway pressure of less than $30 \mathrm{cmH}_{2} \mathrm{O}$ (specific parameter was adjusted according to the type of surgery and patient's condition). Depth of anaesthesia monitoring was completed by bispectral index (BIS) with an appropriate value of 40-60. Before closing the chest, each patient undergoing VATS received a recruitment manoeuvre (RM) by forcing sustained inspiration at the level of $30-40 \mathrm{~cm} \mathrm{H}_{2} \mathrm{O}$ airway pressure for 10 $20 \mathrm{~s}$, and then OLV was converted to TLV until extubation. In addition, a chest tube was connected to a water-sealed bottle to provide drainage of any leaked air or fluid. Those undergoing non-VATS did not receive RM. All patients were transported to the PACU after the operation. Before extubation, the mechanical ventilations in the PACU was the same as that in the operating room. Extubation was performed when the following criteria were met: VT $>5$ $\mathrm{mL} / \mathrm{kg}$, minimal RR of 11 breaths/min, haemodynamic stability (a maximum variation in mean arterial pressure and heart rate was $20 \%$ around the baseline value), normothermia, and TOF $\geq 0.9$. Neostigmine $(0.02 \mathrm{mg} / \mathrm{kg})$ was used for the reversal of neuromuscular blocking before extubation. After extubation, the patient inhaled oxygen through a face mask at 3-6 L/min for approximately $15 \mathrm{~min}$, and then the face masks were removed. Patients were supplemented with oxygen again through masks as temporary treatment if the $\mathrm{SPO}_{2}$ declined to less than $92 \%$.

\section{Lung ultrasound examination}

With a 2 to $5 \mathrm{MHz}$ convex probe in an ultrasound device (Mindray, Guangdong, China), LUS imaging was performed immediately in the PACU by two trained anaesthesiologists (Chen X and Kai S, both with more than 1 year of ultrasound training) once hypoxaemia occurred. The anterior and posterior axillary lines divided each hemithorax into three regions (anterior, lateral and 
posterior), and each region was further divided into two quadrants (superior and inferior) (Fig. 1). The anaesthesiologists performed LUS examination from the left lung to the right in the above order. Atelectasis was diagnosed as a tissue-like pattern or hypoechoic juxta-pleural consolidations with hyperechoic static air bronchograms [10]. A juxta-pleural consolidation or tissue-like structure may also indicate pneumonia. However, the visualization of dynamic air bronchograms helps exclude atelectasis [16]. With a negative predictive value of $100 \%$, the presence of lung sliding excluded the diagnosis of pneumothorax [17]. Moreover, the diagnosis of pneumothorax should be combined with the lung point, barcode sign on $M$ mode and absence of lung sliding $[13,18-20]$. On this basis, the absence of pleural sliding in the anterior, lateral or posterior chest on LUS was defined as small, medium or large pneumothorax [21]. The presence of anechoic area fluctuating with respiration indicated pleural effusion [12]. Examination of pleural effusion was performed with the patient in the semi-recumbent position. A large pleural effusion was diagnosed when the maximal interpleural distance was more than $25 \mathrm{~mm}$ on ultrasonography, and the effusion must be visible in at least three intercostal spaces. Less than $15 \mathrm{~mm}$ of maximal interpleural distance was defined as a small effusion [22]. Combined with symptoms such as dyspnoea, a minimum of $3 \mathrm{~B}$-lines in at least two anterior or lateral quadrants in each thorax may benefit from the consideration of pulmonary oedema [23].

LUS scores ( $0-36$, calculated by summing all 12 individual quadrant scores) are used to assess aeration changes, and a higher grade represents more serious aeration loss but is inapplicable for pneumothorax (Figure 2) [24-26]. The scoring system is as follows: score 0 , healthy lung, equidistant A-lines parallel to the sliding pleura; score 1, moderate aeration loss, no fewer than 3 dispersive $B$ lines originated from the pleural cavity; score 2 , serious aeration loss, presence of coalescent $\mathrm{B}$ lines with pleural irregularities; and score 3, absolute aeration loss, subpleural consolidation. The stored video of the worst irregularity was analysed off-line by Chen X and Kai S. In case of disagreement, a third anaesthesiologist (Lina Y, with 5 years of ultrasound training) reviewed the uncertain images and made the final diagnosis.

\section{Computed tomography scan}

After LUS examination, every patient with stable haemodynamic and spontaneous respiration was transported to the radiology department by a nurse anaesthetist for thoracic CT scan within $1 \mathrm{~h}$ after LUS examination. During transport, all patients received oxygen through face masks. Scanning from the apex to the diaphragm with the patient in the supine position, the examination was performed with a 128-slice spiral CT device (Siemens, Amberg, Germany). With a window width of 1500 Hounsfield units and a section thickness of $0.5 \mathrm{~mm}$, all CT sections were stored for reconstruction and computerized analysis. A trained radiologist blinded to the study reported the CT findings by assessing the absence or presence of consolidation, effusion or pneumothorax as negative (-) or positive $(+)$ in the same anatomic quadrant.

\section{Data collection}

Demographic data, including sex, age, height, weight, American Society of Anesthetist (ASA) score, BMI, vital signs and smoking habit, were recorded. Medical history, pulmonary function test and physical examinations were extracted from the Electronic Medical Record. At the bedside, we collected surgical information, duration of mechanical ventilation and PACU stay, time needed for LUS examination and time needed for CT scan (transportation plus CT scan plus oral report). Cumulative opioid dose (calculated by duration and weight), volume of fluid administration (sum of crystalloid and colloid), blood products and arterial blood gas at the end of the
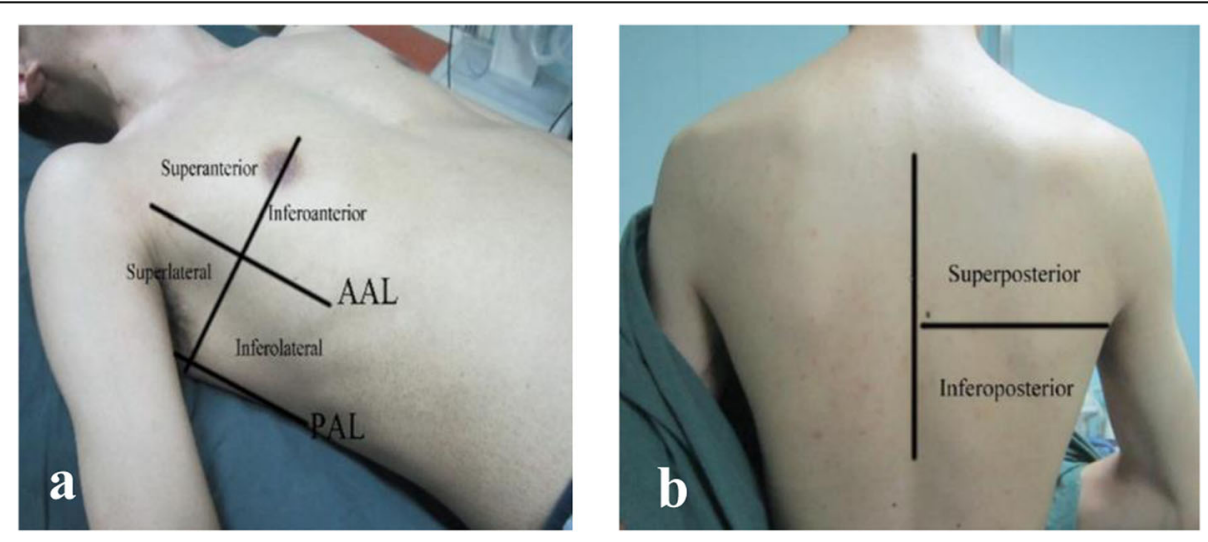

Fig. 1 Hemithorax partition during lung ultrasound examination. a, b Each hemithorax was divided into 6 quadrants by anterior and posterior axillary lines. Abbreviations: AAL, anterior axillary line; PAL, posterior axillary line 

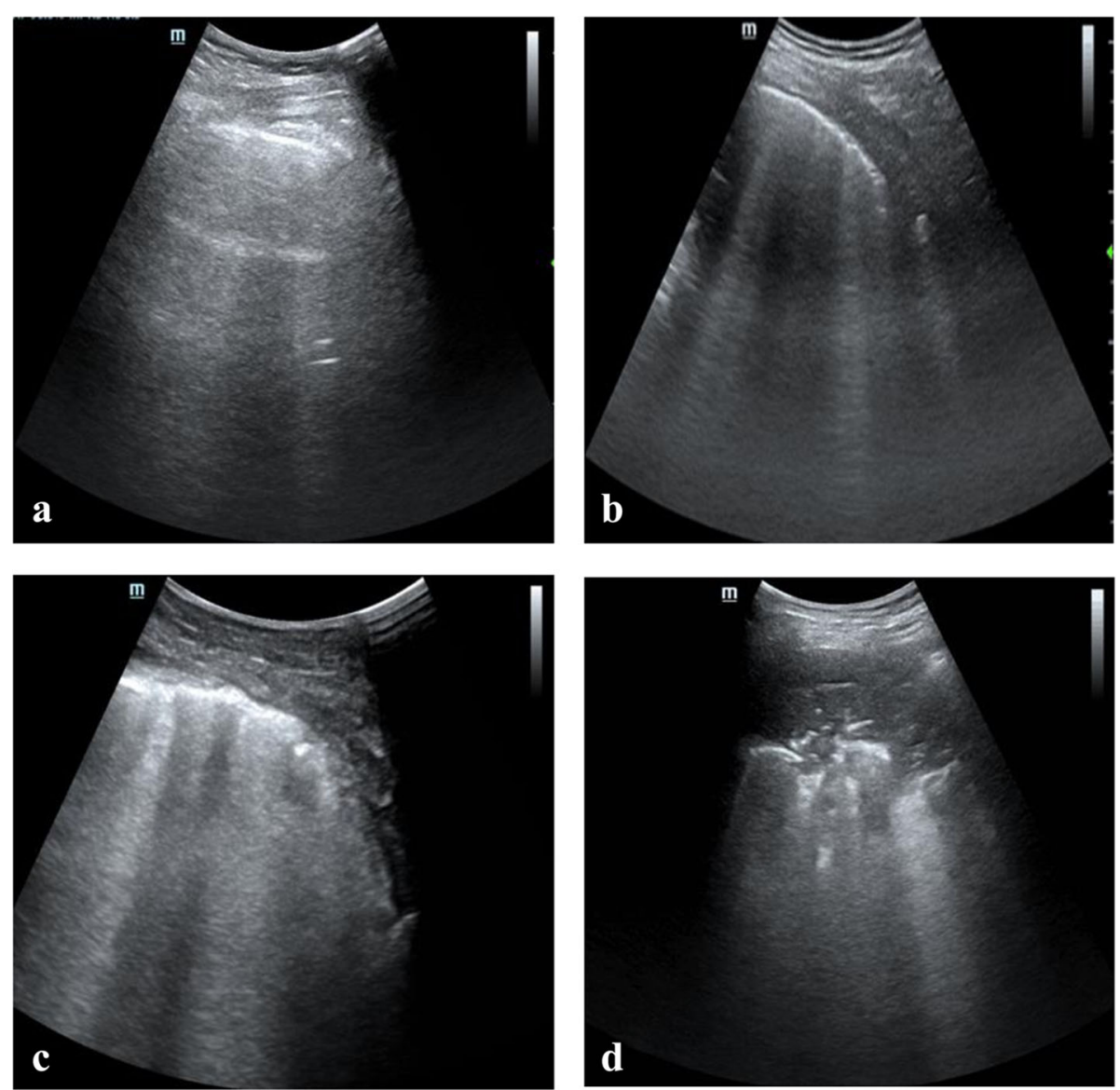

Fig. 2 Lung ultrasound signs with different scores. a score 0, healthy lung, equidistant A-lines parallel to the sliding pleura; b score 1, moderate aeration loss, no fewer than 3 dispersive B lines originated from the pleura; c score 2, serious aeration loss, presence of coalescent B lines with irregular pleura; d, score 3, absolute aeration loss, subpleural consolidation

operation, including haemoglobin, arterial partial pressure of oxygen $\left(\mathrm{PaO}_{2}\right)$, and arterial partial pressure of carbon dioxide $\left(\mathrm{PaCO}_{2}\right)$, were also recorded.

\section{Statistical analysis}

PASS software (version 16.0) was used to calculate the sample size. Assuming the allowable error was $10 \%$ and $\alpha$ was 0.05 (bilateral), on the basis of a previous study, the estimated sensitivity and specificity of LUS were 87.7 and $92.1 \%$, respectively [27]. The calculated sample sizes for sensitivity and specificity were 50 cases and 38 cases, respectively. Considering that the same sample size was adopted for both LUS examination and CT scan, 100 cases were taken from each group of 50 patients. The total sample size was 110 patients when considering a dropout rate of $10 \%$. After testing the normality distribution, the mean \pm standard deviation or median (interquartile range) was used to describe continuous variables, and comparisons were performed with a paired $t$ test or Mann-Whitney U-test as appropriate. Categorical variables are expressed as frequencies and percentages and were compared with the chi- squared test or Fisher's exact test. Spearman's correlation coefficient was used to assess possible factors that may be associated with LUS scores. Correlation coefficient (r) values $<0.3$ indicated nearly no correlation, $r$ values between 0.3 and 0.5 indicated weak correlation, $r$ values between 0.5 and 0.8 indicated moderate correlation and $\mathrm{r}$ values $>0.8$ indicated a high level of correlation. Cohen's kappa was used to test for agreement between the observers. Kappa equal to $0-0.20$ indicated slight agreement, 0.21-0.40 indicated fair agreement, $0.41-0.60$ indicated moderate agreement, $0.61-0.80$ indicated substantial agreement, and 0.81-1 showed almost perfect agreement. SPSS statistical software version 23.0 (IBM Corp, Armonk, NY, USA) was used for data statistics and analysis.

\section{Results}

From January to May 2019, 138 adult patients were evaluated for eligibility. Twenty-five patients (breast operation, haemodynamic instability, etc.) were excluded, and 113 patients were ultimately enrolled (Figure 3). During the study, all the LUS examinations and CT 


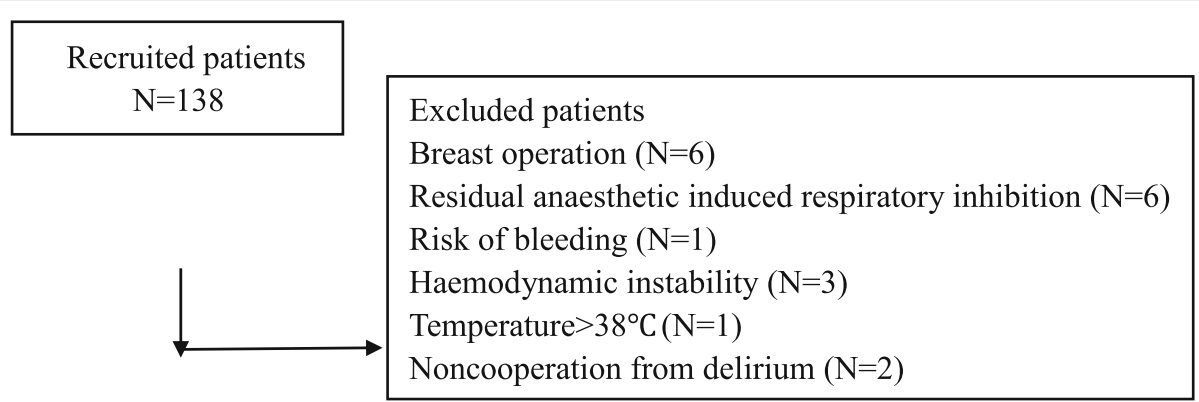

Eligible patients $\mathrm{N}=119$

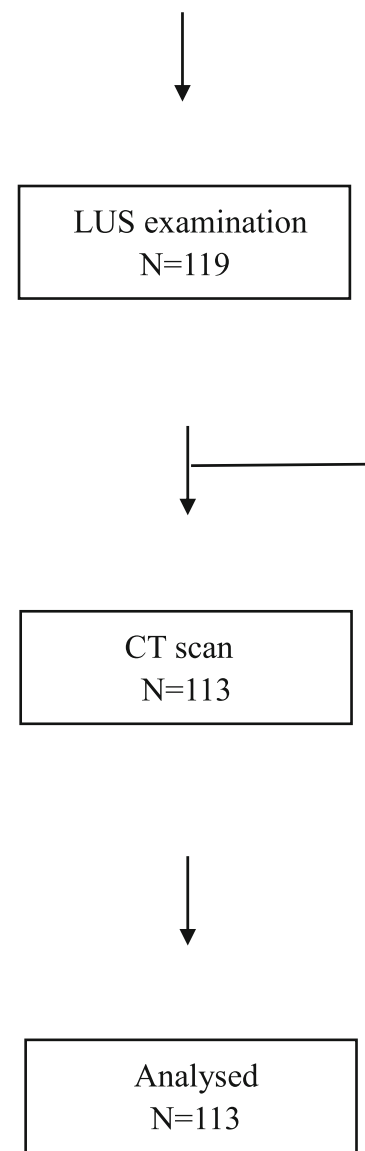

Fig. 3 Flowchart of patient recruitment. Abbreviations: LUS, lung ultrasound; $C$, computed tomography

scans were performed successfully, and a total of 1356 pairs of ultrasound cine-loops and CT images were stored for all patients. Table 1 summarizes the demographic data of the enrolled patients.

Postoperative hypoxaemia in the PACU mainly occurred in patients after abdominal surgery (45 patients, 39.8\%) and VATS (31 patients, 27.4\%), followed by major orthopaedic surgery (17 patients, $15.0 \%)$, neurosurgery (10 patients, $8.8 \%$ ) and other types of surgery (10 patients, 8.8\%). Eighty-two patients (72.6\%) were diagnosed with atelectasis by both CT and LUS. CT scan diagnosed 327 of 1356 quadrants as atelectasis, while LUS revealed the same diagnosis in 311 of the 327 CT-diagnosed quadrants. In patients undergoing nonthoracic surgery, atelectasis 
Table 1 Patient Characteristics ( $N=113)$

\begin{tabular}{ll}
\hline Variables & Mean (SD)/median (IQR) \\
\hline Age $(\mathrm{y})$ & $60.1(12.0)$ \\
Sex, M/F(N) & $61 / 52$ \\
Height (cm) & $164.0(7.5)$ \\
Weight (cm) & $66.1(10.8)$ \\
BMI (kg/m $\left.{ }^{2}\right)$ & $24.5(3.3)$ \\
ASA, $1 / 2 / 3(\mathrm{~N})$ & $7 / 96 / 10$ \\
$\mathrm{SPO}_{2}(\%)$ & $90.0(89.0,91.0)$ \\
Duration of mechanical ventilation (min) & $220.0(162.5,285.0)$ \\
Total infusion (mL) & $1500.0(1000.0,1500.0)$ \\
Duration of PACU stay (min) & $105.0(85.0,137.5)$ \\
LUS score & $13.0(11.0,16.0)$ \\
Smoking status, N (\%) & \\
$\quad$ Current & $10(8.8)$ \\
Previous & $11(9.7)$ \\
Never & $92(81.4)$ \\
Type of surgery, N (\%) & \\
Neurosurgery & $10(8.8)$ \\
Thoracoscopic surgery & $31(27.4)$ \\
Abdominal surgery & $45(39.8)$ \\
Major orthopaedics surgery & $17(15.0)$ \\
Others & $10(8.8)$ \\
\hline
\end{tabular}

Data are described as the mean \pm standard deviation or median and interquartile range, as appropriate

The LUS score was described in patients without pneumothorax $(N=85)$ $\mathrm{SPO}_{2}$ was assessed without inhaled oxygen $15 \mathrm{~min}$ after extubation The duration of PACU stay was the time period from entering the PACU to leaving

Abbreviations: SD Standard deviation; IQR Interquartile range; $M$ male; $F$ Female; BMI Body mass index; ASA American Society of Anesthesiologists classification; $\mathrm{SPO}_{2}$ Oxygen saturation measured by pulse oximetry; PACU Postanaesthesia care unit; LUS Lung ultrasound

was found to be in the posterior zones of both lungs, while the remaining atelectasis was discovered only in the operative lungs of VATS patients. Among the 82 patients with postoperative atelectasis, 19 patients showed signs of atelectasis on preoperative routine chest imaging examinations, while the remaining atelectasis was diagnosed only after surgery.

Twenty-eight patients (24.8\%) (75 quadrants) were diagnosed with pneumothorax by CT scan, whereas 72 quadrants of these 75 quadrants were also diagnosed with pneumothorax with LUS. The majority of these pneumothorax patients (26 patients) were in the VATS group, and the pneumothorax was mainly distributed in anterior and lateral quadrants. In VATS patients, 11 cases of pneumothorax were small, while fifteen were medium in size. The other two patients underwent partial hepatectomy surgery. Regarding the last 2 patients, one was diagnosed with small pneumothorax both by LUS and CT scan, while another was diagnosed with medium pneumothorax, and CT reported an approximate $50 \%$ prevalence of tension pneumothorax.

Pleural effusion was found in 144 quadrants on CT scan in 39 patients (34.5\%), which was primarily exhibited in posterior quadrants. LUS examination detected 131 quadrants with effusion among these CT-diagnosed zones. Nineteen patients (48.7\%) received a diagnosis of pleural effusion on preoperative chest radiographs. The other 20 patients were newly diagnosed in the VATS group, all on the operative sides. One patient was diagnosed with massive pleural effusion on the left side, with visible anechoic effusion in the six quadrants.

One patient was diagnosed with diffuse interstitial syndrome due to multiple B-lines in all 12 lung quadrants, and a CT scan led to the same diagnosis. Both LUS examination and CT scan showed no abnormalities in 12 patients.

The time needed for the LUS examination was significantly shorter than that needed for the CT scan (10.8 \pm $1.8 \mathrm{~min}$ versus $26.8 \pm 4.2 \mathrm{~min}, P<0.001)$. Kappa values for the agreement between the first two observers of atelectasis, pneumothorax and pleural effusion were $0.951(P<0.001), 0.858(P<0.001)$ and $0.964(P<0.001)$, respectively. To resolve this disagreement, the third reviewer was mainly devoted to evaluating the diagnosis of pneumothorax. Table 2 shows the findings of LUS and $\mathrm{CT}$ scans for diagnosing atelectasis, pneumothorax and pleural effusion. LUS was reliable in the diagnosis of atelectasis (with a sensitivity of $98.0 \%$, specificity of $96.7 \%$, positive predictive value of $93.3 \%$, negative predictive value of $99.1 \%$ and diagnostic accuracy of $97.2 \%$ ), pneumothorax (with a sensitivity of $90.0 \%$, specificity of $98.9 \%$, positive predictive value of $96.0 \%$, negative predictive value of $96.9 \%$ and diagnostic accuracy of $96.7 \%$ )

Table 2 Agreement between LUS and CT regarding pulmonary complications in accumulated quadrants

\begin{tabular}{lll}
\hline $\mathrm{CT}$ & LUS & Total \\
\cline { 2 - 3 }+ & - & \\
\hline
\end{tabular}

\begin{tabular}{llcc}
\hline \multicolumn{4}{c}{ a. Agreement between LUS and CT for atelectasis diagnosis } \\
+ & 305 & 22 & 327 \\
- & 6 & 651 & 657 \\
Total & 311 & 673 & 984
\end{tabular}

b. Agreement between LUS and CT for pneumothorax diagnosis

$\begin{array}{llll}+ & 72 & 3 & 75 \\ - & 8 & 253 & 261 \\ \text { Total } & 80 & 256 & 336\end{array}$

c. Agreement between LUS and CT for pleural effusion diagnosis

\begin{tabular}{llll}
+ & 131 & 13 & 144 \\
- & 10 & 314 & 324 \\
Total & 141 & 327 & 468 \\
\hline
\end{tabular}


and pleural effusion (sensitivity of 92.9\%, specificity of $96.0 \%$, positive predictive value of $91.0 \%$, negative predictive value of $96.9 \%$ and diagnostic accuracy of 95.1\%). Among the data we collected, post hoc analyses revealed no correlative factor that significantly influenced LUS scores (Table 3). Postoperative typical LUS and corresponding thoracic CT images of atelectasis, pneumothorax, and pleural effusion are displayed in Figure 4.

\section{Discussion}

Our study showed high accuracy of LUS in diagnosing PPCs such as atelectasis, pneumothorax and pleural effusion, with a high degree of sensitivity and specificity. Consistent with previous publications in both children and adults [28, 29], bedside LUS is reliable, portable, radiationless and fast for the investigation of pulmonary pathologic abnormalities. Previous publications on LUS were mostly from emergency departments and ICUs. To our knowledge, this is the first study to explore the application of LUS to investigate PPCs in patients who developed hypoxaemia in the PACU. In addition, our study population included patients undergoing various types of surgery, and patients with COPD or cardiovascular symptoms were not excluded. This may better reflect the real-world experience. Since postoperative thoracic CT is not routinely used, LUS in the PACU may help differentiate unexpected respiratory pathologies. Most likely, our study could provide clinical significance for the timely and appropriate treatment of postoperative hypoxaemia in the future.

PPCs are common after general anaesthesia, while hypoxaemia is primary triggered by atelectasis from compression, gas absorption and loss of surfactant [30]. Postoperative atelectasis was associated with pneumonia and could result in delayed discharge [31]. Early detection and treatment of atelectasis is essential for improving prognosis. Due to advantages such as simplicity, convenience, time requirement and non-radiation, LUS can be performed multiple times at the bedside. The sensitivity and specificity of the diagnosis of atelectasis by lung pulse in ultrasound were 93 and 100\%, respectively $[32,33]$. When compared with magnetic resonance imaging (MRI), LUS showed a sensitivity of $88 \%$, specificity of $89 \%$ and accuracy of $88 \%$ in diagnosing pulmonary atelectasis [10]. LUS demonstrated excellent diagnostic accuracy (97.2\%) in our study, which was higher than that reported (90.7\%) by $\mathrm{Yu} \mathrm{X}$ et al. [27] Yu's study only enrolled patients undergoing elective intracranial surgery, and those with preoperative pulmonary comorbidities were excluded, whereas our study included a heterogeneous patient population for diversity. To eliminate the interference of the adipose layer in the ultrasonic image, obese patients $\left(B M I>40 \mathrm{~kg} / \mathrm{m}^{2}\right.$ ) were excluded. Considering the safety risk of transferring patients to CT scans, those with haemodynamic instability were also excluded. Hypoxaemia might also frequently occur in these patients, but the whole study only excluded 3 relevant patients (Figure 3), and this exclusion exerted almost no effect on the result. The incidence of postoperative atelectasis (67.2\%) in our study was lower than that previously reported (90\%) [34], which was partly due to routine recruitment manoeuvres at the end of the VATS group. Through lung-protective strategies such as low TV, a lower $\mathrm{FiO}_{2}$, higher RRRM and PEEP have been reported to significantly decrease postoperative respiratory complications [35-37] and were applied in our anaesthesia protocol. However, atelectasis still occurred frequently. PEEP has been reported as a successful method for improving oxygenation and respiratory function during general anaesthesia, but the optimal level is still inconclusive [38-40]. Although a PEEP of $5 \mathrm{cmH}_{2} \mathrm{O}$ in our study has been reported in a previous study, a higher PEEP may be much more beneficial for reducing atelectasis formation, as has been

Table 3 Correlation between possible factors and lung ultrasound scores ( $N=85)$

\begin{tabular}{lll}
\hline Variables & Correlation Coefficient $(\mathrm{r})$ & $P$ \\
\hline $\mathrm{Sex}(\mathrm{M} / \mathrm{F})$ & 0.229 & 0.035 \\
$\mathrm{Age}(\mathrm{y})$ & -0.041 & 0.707 \\
$\mathrm{BMI}\left(\mathrm{kg} / \mathrm{m}^{2}\right)$ & -0.127 & 0.246 \\
$\mathrm{SPO}_{2}(\%)$ & -0.244 & 0.024 \\
Smoking & -0.039 & 0.725 \\
Duration of mechanical ventilation (min) & -0.127 & 0.245 \\
Type of surgery & 0.075 & 0.494 \\
Sufentanil dose $(\mathrm{ug} / \mathrm{kg} / \mathrm{h})$ & 0.125 & 0.253 \\
Total infusion $(\mathrm{mL})$ & 0.046 & 0.677 \\
Transfusion $(\mathrm{Y} / \mathrm{N})$ & -0.156 & 0.155 \\
\hline
\end{tabular}

Lung ultrasound scores were recorded and analysed in patients without pneumothorax

Abbreviations: LUS Lung ultrasound; $M$ Male; $F$ Female; $B M I$ Body mass index; $\mathrm{SPO}_{2}$ Oxygen saturation measured by pulse oximetry 

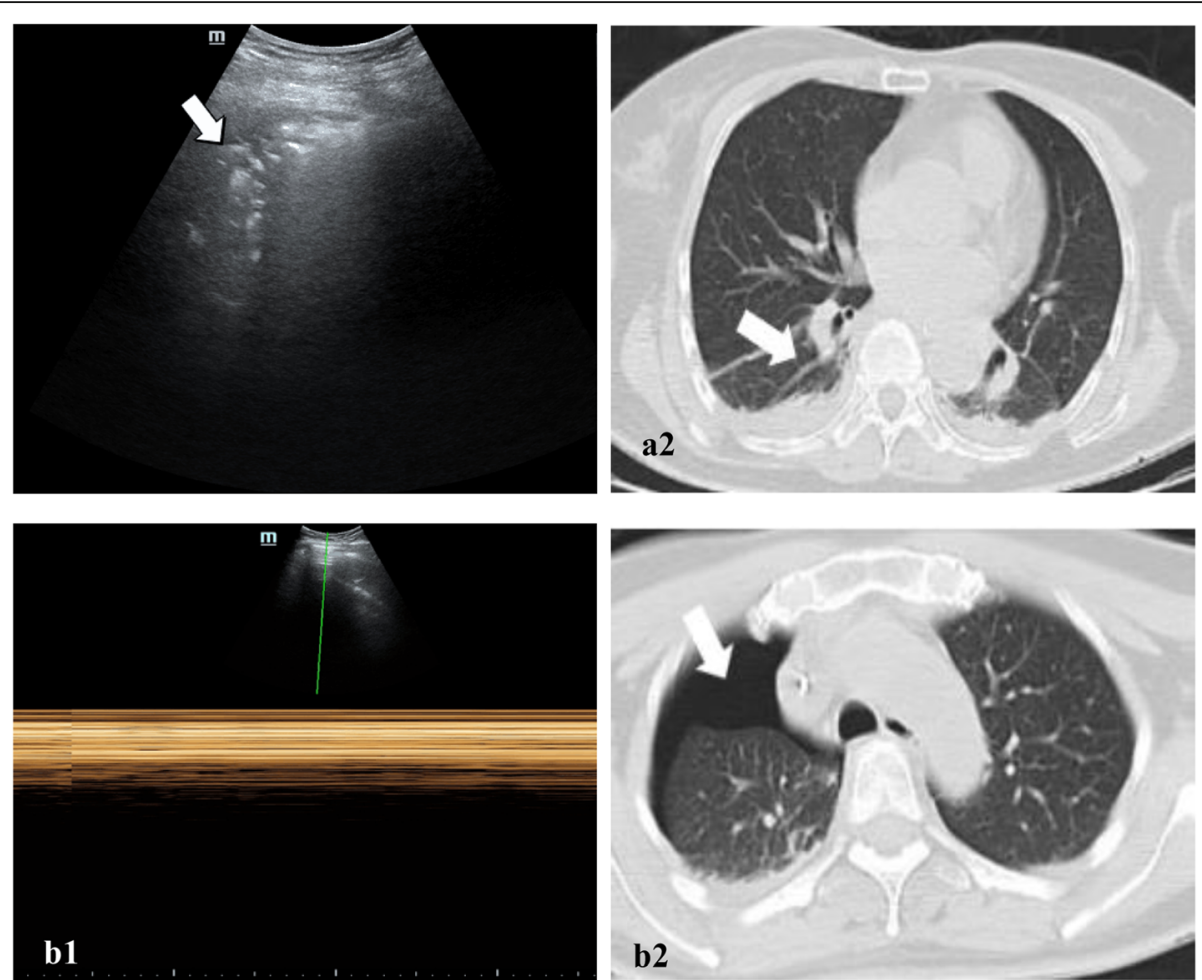

b1

\section{b2}
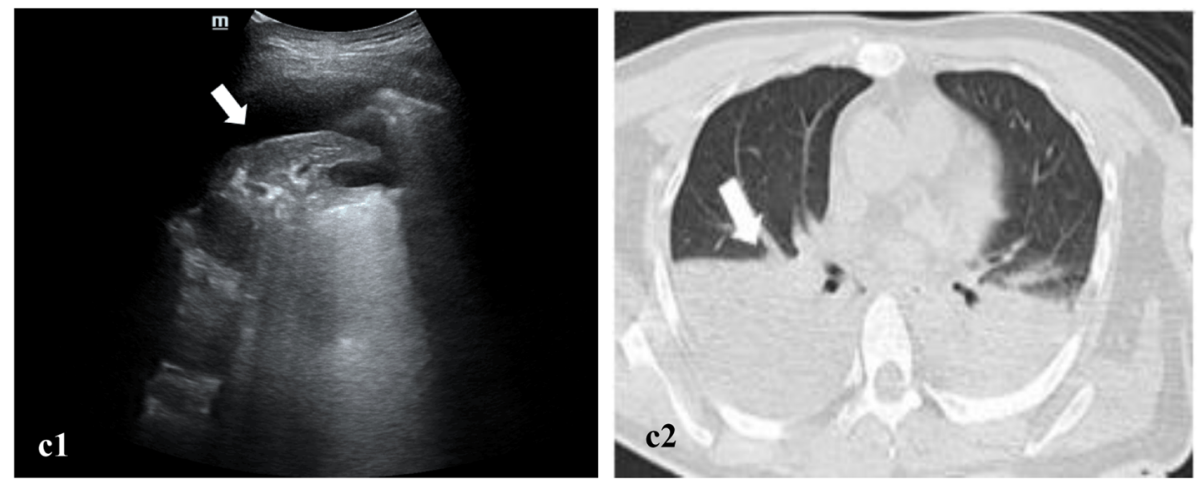

Fig. 4 Typical pulmonary pathologies on both LUS and thoracic CT in the same regions. (a1) Typical LUS signs of atelectasis in the dorsal quadrant of the lung presented as tissue-like patterns (left, white arrow), (a2) CT signs of corresponding regions presented as a crescent shape (right, white arrow)(b1) Typical LUS on M-mode of pneumothorax in the anterior quadrant of the lung presented as a bar code sign, (b1) CT signs of corresponding regions presented as very-low-density gas window (right, white arrow).(c1) Typical LUS of pleural effusion in the dorsal quadrant of the lung presented as anechoic area (left, white arrow), (c2) CT signs of corresponding regions presented as a half-moon (right, white arrow).Abbreviations: LUS, lung ultrasound; CT, computed tomography.

recommended by some researchers [41]. RM combined with PEEP was also beneficial for reducing atelectrauma [42], but it was only performed in OLV in our study. This may explain the high occurrence of atelectasis in the non-VATS group. To date, the optimal systematization of RM remains a matter of debate, as findings have identified a potential danger of excessive RM during OLV, resulting in increased mortality [43]. Under perioperative ultrasoundguided recruitment manoeuvres and moderate PEEP, the incidence of atelectasis and postoperative hypoxaemia decreased in both infants and paediatric cardiac patients [41, 44, 45].
Detecting the effects of different levels of PEEP and RM on PPCs by lung ultrasound still needs more research.

Similar to Xirouchaki et al.'s findings, our study showed that LUS was effective in the diagnosis of pneumothorax [46]. The absence of lung sliding or Bline to diagnose pneumothorax by LUS has a sensitivity of 88 to $100 \%$ [47, 48]. Our study further confirmed the $90 \%$ sensitivity of LUS to diagnose pneumothorax. Because the thorax was opened for VATS, the pneumothorax was deemed residual gas. Patella et al. [47, 49] demonstrated that LUS could also effectively and 
accurately evaluate the small amount of pneumothorax remaining after thoracic drainage, which was faster and more accurate than CXR. Senniappan's study suggested that LUS may be an alternative to CXR for the follow-up of pneumothorax due to its superior sensitivity, portability and reduction in radiation exposure [50]. Wei et al. [51] advocated for the use of daily LUS in the postoperative period to enhance recovery after thoracic surgery. Similar to prior publications [52-54], we demonstrated that LUS was sensitive and specific to diagnose pleural effusion with added benefits of convenience and safety. Compared with traditional methods, placing the thorax tube for fluid drainage under ultrasound guidance is safer and more effective and can reduce the incidence of pneumothorax [55]. The severity of hypoxaemia depended on effusion size and the patient's cardiopulmonary condition, while prompt diagnosis of pleural effusion is vital to evaluate the optimal therapeutic choice. Effusion drainage ultrasound guidance would relieve compression atelectasis of the adjacent lung and improve respiratory mechanics and oxygenation.

Although LUS is an operator-dependent skill and adequate training is needed for effective clinical usage, it can be readily learned with a very simple device. See et al. [56] confirmed that after only $3 \mathrm{~h}$ of lung ultrasound self-study and an average of 15 scans of patients, the accuracy of diagnosis in trainees with no prior ultrasound experience would achieve $95.4 \%$, and the medial scanning duration was only $12 \mathrm{~min}$. In Zhan's study, the implementer was a paediatric resident with no expert supervision and minimal practical ultrasound experience, and this examiner completed the LUS examinations accurately [11]. Compared to experts, inexperienced resident physicians in emergency medicine with 30 min LUS training can effectively identify B-lines with more than $80 \%$ sensitivity and specificity [57]. After brief training, surgical residents or medical students could also perform LUS well and interpret the results accurately [12]. To improve diagnostic reliability, ultrasound was performed and evaluated by two researchers with long-term training in our study. The results showed high agreement between the two observers, while the disagreement was mainly in regard to pneumothorax, probably because the diagnosis of pneumothorax by ultrasound should be based on multiple signs.

There are several limitations of our study. First, the 1-h time interval between LUS examination and CT scan and the suction impact of the water seal bottle may create false-positive results, while the obstruction of ultrasound views by the scapula and ribs could introduce falsenegative results. Second, the presence of consolidation on LUS alone was insufficient for diagnosing pneumonia. In a recent study by Zhou et al. [58], the combination of LUS and procalcitonin had a better diagnostic value for pneumonia. Timely diagnosis of suspected aspiration pneumonia by LUS intraoperatively may be beneficial for patients but still needs more research in our future work. Third, different ventilatory management strategies may have different effects, especially on thoracic surgery, whereas the inclusion of multiple types of surgery patients was a limitation in our study. Although RM did not affect the accuracy of the comparison between LUS and CT, performing a subgroup analysis of this population might be meaningful. To refine our research, we conducted an LUS-related study specifically on thoracic surgery. Last, because of the BMI of the population in our study, our findings might not reflect the sensitivity and specificity of LUS to diagnose PPCs in patients with higher BMI, as those with a BMI $>40 \mathrm{~kg} / \mathrm{m}^{2}$ were excluded.

\section{Conclusions}

In conclusion, we showed that the application of LUS to diagnose the aetiologies of hypoxaemia in healthy-weight PACU patients is feasible and quick. LUS was sensitive and specific to diagnose PPCs when compared to the sensitivity and specificity of thoracic CT scans.

\section{Abbreviations \\ PPCs: Postoperative pulmonary complications; CRX: Chest x-rays; $C T$ : Computed tomography; LUS: Lung ultrasound; PACU: Postanesthesia care unit; $\mathrm{SPO}_{2}$ : Pulse oximetry; BMl: Body mass index; TOF: Train of four stimulation; ICU: Intensive care unit; $\mathrm{FiO}_{2}$ : Inspiration oxygen fraction; OLV: One-lung ventilation; VATS: Video-assisted thoracoscopic surgery; TLV: Two-lung ventilation; RR: Respiratory rate; PEEP: Positive end-expiratory pressure; $\mathrm{P}_{\mathrm{ET}} \mathrm{CO}_{2}$ : End-tidal carbon dioxide pressure; BIS: Bispectral index; RM: Recruitment maneuver; ASA: American society of anesthetist; $\mathrm{PaO}_{2}$ : Arterial partial pressure of oxygen; $\mathrm{PaCO}_{2}$ : Arterial partial pressure of carbon dioxide; MRI: Magnetic resonance imaging}

\section{Acknowledgments}

The authors would like to thank Gundappa Neelakanta M.B., B.S. (Ronald Reagan UCLA Medical Center Department of Anesthesiology \& Perioperative Medicine, USA) for his valuable guidance for article writing.

\section{Authors' contributions}

Name: CX, Contribution: made substantial contributions to the conception and design of the work; the acquisition, analysis, interpretation of data; drafted the work and substantively revised it. Name: KS, Contribution: made substantial contributions to the conception and design of the work; the acquisition, analysis, interpretation of data; the creation of new software used in the work; drafted the work. Name: YY, Contribution: made substantial contributions to the conception and design of the work; the acquisition, analysis, interpretation of data; the creation of new software used in the work; Name: YM, Contribution: made substantial contributions to the conception and design of the work; the acquisition, analysis, interpretation of data. Name: XY, Contribution: made substantial contributions to the conception and design of the work. Name: LY, Contribution: made substantial contributions to the conception and design of the work; the creation of new software used in the work. Name: $\mathrm{HH}_{\text {, }}$ Contribution: the acquisition, analysis, interpretation of data; the creation of new software used in the work; drafted the work and substantively revised it. Name: MY, Contribution: made substantial contributions to the conception and design of the work; drafted the work and substantively revised it. All authors have read and approved the final manuscript. 


\section{Availability of data and materials}

The datasets generated and/or analysed during the current study are not publicly available due to the manuscript has not been received yet but are available from the corresponding author on reasonable request.

\section{Ethics approval and consent to participate}

The study was approved by the review committee of Second Affiliated Hospital of Zhejiang University (IR2018001133, 2018/12/05) and registered at ClinicalTrials.gov (NCT03802175) before patient enrollment. Informed consents were obtained from all patients.

\section{Consent for publication}

Not applicable.

\section{Competing interests}

The authors declare that they have no competing interests.

\section{Author details}

${ }^{1}$ Department of Anesthesiology and Pain Medicine, the Second Affiliated Hospital, School of Medicine, Zhejiang University, Jiefang Road 88th, Hangzhou 310016, People's Republic of China. ${ }^{2}$ Department of Anesthesiology \& Perioperative Medicine, University of Louisville, Louisville, KY 40202, USA. ${ }^{3}$ Department of Anesthesiology and Pain Medicine, Second Affiliated Hospital, Zhejiang University School of Medicine, 88 Jiefang Road, Hangzhou 310009, NO, China.

\section{Received: 23 April 2020 Accepted: 9 August 2020}

Published online: 01 September 2020

\section{References}

1. Kim TH, Lee JS, Lee SW, Oh YM. Pulmonary complications after abdominal surgery in patients with mild-to-moderate chronic obstructive pulmonary disease. Int J Chron Obstruct Pulmon Dis. 2016;11:2785-96.

2. Sun Z, Sessler DI, Dalton JE, et al. Postoperative hypoxemia is common and persistent: a prospective blinded observational study. Anesth Analg. 2015; 121:709-15.

3. Belcher AW, Khanna AK, Leung S, et al. Long-acting patient-controlled opioids are not associated with more postoperative hypoxemia than shortacting patient-controlled opioids after noncardiac surgery: a cohort analysis. Anesth Analg. 2016;123:1471-9.

4. L'her $E$, Jaber $\mathrm{S}$, Jacob C, et al. Automated oxygen administration versus conventional oxygen therapy after major abdominal or thoracic surgery: study protocol for an international multicentre randomised controlled study. BMJ Open. 2019;9:e023833.

5. Govinda R, Kasuya Y, Bala E, et al. Early postoperative subcutaneous tissue oxygen predicts surgical site infection. Anesth Analg. 2010;111:946-52.

6. Purhonen S, Niskanen M, Wüstefeld M, Mustonen P, Hynynen M. Supplemental oxygen for prevention of nausea and vomiting after breast surgery. Br J Anaesth. 2003;91:284-7.

7. Bass JL, Corwin M, Gozal D, et al. The effect of chronic or intermittent hypoxia on cognition in childhood: a review of the evidence. Pediatrics. 2004;114:805-16.

8. Chiappetta M, Meacci E, Cesario A, et al. Postoperative chest ultrasound findings and effectiveness after thoracic surgery: a pilot study. Ultrasound Med Biol. 2018:44:1960-7.

9. Mojoli F, Bouhemad B, Mongodi S, Lichtenstein D. Lung ultrasound for critically ill patients. Am J Respir Crit Care Med. 2019;199:701-14.

10. Acosta C, Maidana G, Jacovitti D, et al. Accuracy of transthoracic lung ultrasound for diagnosing anesthesia-induced atelectasis in children. Anesthesiology. 2014;120:1370-9.

11. Zhan C, Grundtvig N, Klug B. Performance of bedside lung ultrasound by a pediatric resident: a useful diagnostic tool in children with suspected pneumonia. Pediatr Emerg Care. 2018;34:618-22.

12. Steinmetz P, Oleskevich S, Dyachenko A, McCusker J, Lewis J. Accuracy of medical students in detecting pleural effusion using lung ultrasound as an adjunct to the physical examination. J Ultrasound Med. 2018;37:2545-52.

13. Alrajhi K, Woo MY, Vaillancourt C. Test characteristics of ultrasonography for the detection of pneumothorax: a systematic review and meta-analysis. Chest. 2012;141:703-8.

14. Bouhemad B, Mongodi S, Via G, Rouquette I. Ultrasound for "lung monitoring" of ventilated patients. Anesthesiology. 2015;122:437-47.
15. Russell GB, Graybeal JM. Hypoxemic episodes of patients in a postanesthesia care unit. Chest. 1993;104:899-903.

16. Bouhemad B, Dransart-Rayé O, Mojoli F, Mongodi S. Lung ultrasound for diagnosis and monitoring of ventilator-associated pneumonia. Ann transl med. 2018;6:418.

17. Husain LF, Hagopian L, Wayman D, Baker WE, Carmody KA. Sonographic diagnosis of pneumothorax. J Emerg Trauma Shock. 2012;5:76-81.

18. Santos-Silva J, Lichtenstein D, Tuinman PR, Elbers PWG. The lung point, still a sign specific to pneumothorax. Intensive Care Med. 2019;45:1327-8.

19. Williamson JP, Grainge C, Parameswaran A, Twaddell SH. Thoracic ultrasound: what non-radiologists need to know. Curr pulmonol rep. 2017;6:39-47.

20. Maury E, Pichereau C, Bourcier S, et al. Diagnostic ultrasound in pneumothorax. Rev Mal Respir. 2016;33:682-91.

21. Zhang M, Liu Z, Yang J, et al. Rapid detection of pneumothorax by ultrasonography in patients with multiple trauma. Crit Care. 2006;10:R112.

22. Razazi K, Boissier F, Neuville M, et al. Pleural effusion during weaning from mechanical ventilation: a prospective observational multicenter study. Ann Intensive Care. 2018;8(1):103.

23. Wooten WM, Shaffer LET, Hamilton LA. Bedside ultrasound versus chest radiography for detection of pulmonary edema: a prospective cohort study. Ultrasound Med. 2018;38:967-73.

24. Markarian T, Zieleskiewicz L, Perrin G, et al. A lung ultrasound score for early triage of elderly patients with acute dyspnea. Cjem. 2019;21:399-405.

25. Yin W, Zou T, Qin Y, et al. Poor lung ultrasound score in shock patients admitted to the ICU is associated with worse outcome. BMC Pulm Med. 2019;19:1.

26. Frassi F, Gargani L, Gligorova S, Ciampi Q, Mottola G, Picano E. Clinical and echocardiographic determinants of ultrasound lung comets. Eur J Echocardiogr. 2007:8:474-9.

27. Yu X, Zhai Z, Zhao Y, et al. Performance of lung ultrasound in detecting Peri-operative atelectasis after general anesthesia. Ultrasound Med Biol. 2016:42:2775-84.

28. Blank DA, Kamlin COF, Rogerson SR, et al. Lung ultrasound immediately after birth to describe normal neonatal transition: an observational study. Arch Dis Child Fetal Neonatal Ed. 2018;103:F157-62.

29. Lui JK, Banauch Gl. Diagnostic bedside ultrasonography for acute respiratory failure and severe hypoxemia in the medical intensive care unit: basics and comprehensive approaches. J Intensive Care Med. 2017;32:355-72.

30. Magnusson L, Spahn DR. New concepts of atelectasis during general anaesthesia. Br J Anaesth. 2003;91:61-72.

31. Brooks-Brunn JA. Postoperative atelectasis and pneumonia. Am J Crit Care. 1995:4:340-9.

32. Lichtenstein D, Meziere G, Seitz J. The dynamic air bronchogram. A lung ultrasound sign of alveolar consolidation ruling out atelectasis. Chest. 2009; 135:1421-5.

33. Blancas Gomez-Casero R, Alonso Fernandez MA, Ballesteros Ortega D, Martinez GO. Subpleural artifact in lung pulse evidenced by M-mode ultrasound in a patient with atelectasis. Rev Esp Anestesiol Reanim. 2016;63:127.

34. Tusman G, Böhm S, Warner DO, Sprung J. Atelectasis and perioperative pulmonary complications in high-risk patients. Curr Opin Anaesthesiol. 2012; 25:1-10.

35. Licker M, Diaper J, Villiger $Y$, et al. Impact of intraoperative lung-protective interventions in patients undergoing lung cancer surgery. Crit Care. 2009;13:R41.

36. Borges J, Amato M, Hedenstierna G. The increasing call for protective ventilation during anesthesia. JAMA Surg. 2017;152:893-4.

37. Campos JH, Feider A. Hypoxia during one-lung ventilation-a review and update. J Cardiothorac Vasc Anesth. 2018;32:2330-8.

38. Chalhoub V, Yazigi A, Sleilaty $G$, et al. Effect of vital capacity manoeuvres on arterial oxygenation in morbidly obese patients undergoing open bariatric surgery. Eur J Anaesthesiol. 2007;24:283-8.

39. Ferrando C, Soro M, Unzueta C, et al. Individualised perioperative open-lung approach versus standard protective ventilation in abdominal surgery (iPROVE): a randomised controlled trial. Lancet Respir Med. 2018;6:193-203.

40. Bluth T, Serpa Neto A, Schultz MJ, Pelosi P, Gama de Abreu M. Effect of intraoperative high positive end-expiratory pressure (PEEP) with recruitment maneuvers vs low PEEP on postoperative pulmonary complications in obese patients. Jama. 2019;321:2292.

41. Östberg E, Thorisson A, Enlund M, Zetterström H, Hedenstierna G, Edmark L. Positive end-expiratory pressure alone minimizes atelectasis formation in nonabdominal surgery: a randomized controlled trial. Anesthesiology. 2018; 128:1117-24. 
42. van der Zee P, Gommers D. Recruitment maneuvers and higher PEEP, the socalled open lung concept, in patients with ARDS. Critical Care. 2019;23:73.

43. Kidane B, Palma DC, Badner NH, et al. The potential dangers of recruitment maneuvers during one lung ventilation surgery. J Surg Res. 2019;234:178-83.

44. Song IK, Kim EH, Lee JH, Ro S, Kim HS, Kim JT. Effects of an alveolar recruitment manoeuvre guided by lung ultrasound on anaesthesia-induced atelectasis in infants: a randomised, controlled trial. Anaesthesia. 2017;72: 214-22.

45. Song IK, Kim EH, Lee JH, Kang P, Kim HS, Kim JT. Utility of perioperative lung ultrasound in pediatric cardiac surgery: a randomized controlled trial. Anesthesiology. 2018;128:718-27.

46. Xirouchaki N, Magkanas E, Vaporidi K, et al. Lung ultrasound in critically ill patients: comparison with bedside chest radiography. Intensive Care Med. 2011;37:1488-93.

47. Patella M, Saporito A, Puligheddu C, et al. Lung ultrasound to detect residual pneumothorax after chest drain removal in lung resections. Ann Thorac Surg. 2018;105:1537-42.

48. Ding W, Shen Y, Yang J, He X, Zhang M. Diagnosis of pneumothorax by radiography and ultrasonography: a meta-analysis. Chest. 2011;140:859-66.

49. Galbois A, Ait-Oufella H, Baudel JL, et al. Pleural ultrasound compared with chest radiographic detection of pneumothorax resolution after drainage. Chest. 2010;138:648-55.

50. Senniappan K, Sreedhar R, Babu MSS, et al. Bedside lung ultrasound for postoperative lung conditions in cardiothoracic intensive care unit: diagnostic value and comparison with bedside chest roentgenogram. Anesth Essays Res. 2019;13:649-53.

51. Wei X, Li S, Cheng S, Qiu L, Che G. Does daily chest ultrasound in the postoperative period contribute to an enhanced recovery after surgery pathway for patients undergoing general thoracic surgery? J Thorac Dis. 2019;11(Suppl 9):1246-9.

52. Kurian J, Levin TL, Han BK, Taragin BH, Weinstein S. Comparison of ultrasound and $C T$ in the evaluation of pneumonia complicated by parapneumonic effusion in children. Am J Roentgenol. 2009;193:1648-54.

53. Lichtenstein D, Goldstein I, Mourgeon E, Cluzel P, Grenier P, Rouby JJ. Comparative diagnostic performances of auscultation, chest radiography, and lung ultrasonography in acute respiratory distress syndrome. Anesthesiology. 2004;100:9-15.

54. Volpicelli G, Elbarbary M, Blaivas M, et al. International evidence-based recommendations for point-of-care lung ultrasound. Intensive Care Med. 2012;38:577-91.

55. Perazzo A, Gatto P, Barlascini C, Ferrari-Bravo M, Nicolini A. Can ultrasound guidance reduce the risk of pneumothorax following thoracentesis? J Bras Pneumol. 2014;40:6-12.

56. See KC, Ong V, Wong SH, et al. Lung ultrasound training: curriculum implementation and learning trajectory among respiratory therapists. Intensive Care Med. 2015;42:63-71.

57. Chiem AT, Chan CH, Ander DS, Kobylivker AN, Manson WC, Costantino T. Comparison of expert and novice sonographers' performance in focused lung ultrasonography in dyspnea (FLUID) to diagnose patients with acute heart failure syndrome. Acad Emerg Med. 2015;22:564-73.

58. Zhou J, Song J, Gong S, et al. Lung ultrasound combined with Procalcitonin for a diagnosis of ventilator-associated pneumonia. Respir Care. 2019;64: 519-27.

\section{Publisher's Note}

Springer Nature remains neutral with regard to jurisdictional claims in published maps and institutional affiliations.

Ready to submit your research? Choose BMC and benefit from:

- fast, convenient online submission

- thorough peer review by experienced researchers in your field

- rapid publication on acceptance

- support for research data, including large and complex data types

- gold Open Access which fosters wider collaboration and increased citations

- maximum visibility for your research: over $100 \mathrm{M}$ website views per year

At BMC, research is always in progress.

Learn more biomedcentral.com/submissions 